\title{
Extracting Interaction Effect of Cognitive Task and Unwanted Cognitive Activity in Pre-Sleep Period
}

\author{
Chuang Gao,Zongkui Zhou \\ Key Laboratory of Adolescent Cyberpsychology and \\ Behavior \\ Central China Normal University \\ Ministry of Education \\ Wuhan 430079, P.R.China
}

\author{
Wei Wei \\ College of basic research \\ Central China Agriculture University \\ Wuhan, China.
}

Genghu Shi, Huiyong Hu, Rui Jiang
School of psychology
Central China Normal University
Wuhan 430079, Hubei Province, China

\begin{abstract}
The difficulty of switching off unwanted pre-sleep cognitive activities always causes most people with insomnia unable to fall asleep. Previous studies suggested that manipulating pre-sleep thoughts with cognitive task could change sleep onset latency. The interaction effect of cognitive task and unwanted thoughts is a mixed effect in which each mechanism alone is not completely understood. The present study tries to explore the mixed effect and extract the single effect from it.

Seven people with insomnia were investigated and the general cognitive task controlled internally by insomniacs themselves was replaced with an audio task in which the participants have to react distinctively to two different auditory stimuli. The reaction time for which reflected the interaction effect was recorded and then analyzed by the autocorrelation and partial autocorrelation method. A general decline tendency which is called decline effect was observed in the single effect and for most insomniacs the length of the decline effect was around 4 or 5 seconds. In addition, it indicated that such decline effect could be a potential candidate for investing the correlation between cognitive load and interaction effect.
\end{abstract}

Keywords-insomnia; cognitive; autocorrelation; partial autocorrelation

\section{INTRODUCTION}

The difficulty of switching off unwanted pre-sleep cognitive activities always causes most insomniacs unable to fall asleep [1-7]. In previous studies of insomnia, accumulated evidences suggest that the manipulation of presleep thoughts with cognitive task such as mental arithmetic or imagery distraction could lead to sleep onset latency change [1, 8-12].

According to the account that the cognitive space is limited, the cognitive task will occupy sufficient cognitive space to keep the individual from re-engaging in the personally relevant negative intrusive thought. So, the adoption of a cognitive task can interrupt and suppress the unwanted thought $[11,13-16]$. And then the problem of long sleep onset latency[17] will be solved for the maintenance of suppression for intrusive thought[15].

The maintenance of suppression suggests that there is continuous cognitive interaction between the cognitive task and the intrusive thought. That is to say, the repeated cognitive task is needed to maintain the continuous suppress effect. The cognitive interaction effect at one time point will decay but not vanish immediately. This indicates that the current cognitive interaction effect will mix with the previous effects. So, it is the complex mixed effect that has impact on intrusive thought. The previous cognitive interaction effect which mixes with current effect is called decline effect. Accordingly, detecting cognitive interaction dynamically in real-time and extracting the mixed effect and decline effect are likely to be crucial to understanding the cognitive interaction and suppression of the unwanted thought for the insomnias in pre-sleep period.

Therefore, in the present study we will use an audio cognitive task to manipulate pre-sleep cognitive activities by which the insomniacs can make behavioral response as the stimuli presented and the behavioral response magnitude (such as reaction time) can be recorded.

This task is chosen for two main reasons. Primarily, it's a valid behavioral dynamic measurement of subjective information in real-time and easy to distinguish sleep and pre-sleep. So it is often used to detect cognitive processing in sleep onset[18-25]. Secondly, though the internal control strategy such as mental arithmetic or imagery distraction is replaced by the external control strategy such as audio task, the external task will also interact with pre-sleep unwanted thought, and the cognitive space occupation hypothesis would be satisfied. So the interaction information will be included in the behavioral magnitude. By repeated measuring, series of interaction data can be easily got.

For the decline effect, the data of behavioral response time-series is dependent. By calculating the values of autocorrelations and partial autocorrelations we can assess 
the value of the dependency. And based on the changes of dependency, we try to evaluate decline effect and mixed effect in this paper.

\section{METHOD}

\section{A. Participants}

The volunteers were recruited from Central China Normal University and they all described their sleep quality as "poor". The criterion for inclusion in this study was a total score of more than seven on the Pittsburg Sleep Quality Index (PSQI)[26, 27]. After PSQI approval and informed written consent, seven volunteers who had not received pharmacologic sleep aids were enrolled.

\section{B. Procedures}

All participants spent four consecutive nights, each night held one session in a sound proof sleep room with airconditioned. And the first night was considered as adaptation period. Each subject was asked to come to the sleeping room one hour earlier before their bedtime routine.

For the first night after informed written consent, the participants received the instruction on the experimental procedures. They were asked to perform a sound discrimination task in which there were two short audio stimuli. One was clearly such as dropping water, the other was relaxing. When they heard the clear tone they should click the right button of a mouse and the relaxing one with the left button.

The intensity of the tone was, prior to the beginning of sleep testing session, adjusted to the lowest level that subjects could consistently register while awake. The interstimulus interval (ISI) (see Figure $1 \mathrm{a}$ ) was constant during the same testing session in order to satisfy with time series analysis. During the first two night testing session, ISI was 2 seconds and 3, 4 or 5 seconds was chosen in following days. Matlab7.0 with a psychtoolbox3.0[28] was used to generate audio tone and record the timing of both the tone and the mouse-click responses.

The formal test session was begun when insomniacs reported sleepy. Insomniacs were then placed in the dark sleep room and instructed to fall asleep. There were powered speakers and a mouse connected with the computer which was outside the testing room (see Figure $1 \mathrm{~b}$ ). The testing was over when subject consecutively lost responses in $30 \mathrm{~min}$. After testing session, a subjective assessment of sleep quality was made. Insomniacs were interviewed to report their subjective sleep quality.

\section{Data Coding}

The insomniacs' behavioral response magnitude of reaction time in the same testing session is approximately time series if the stimuli were set to occur in constant intervals. Then we used $x$ to represent the observation value in the data
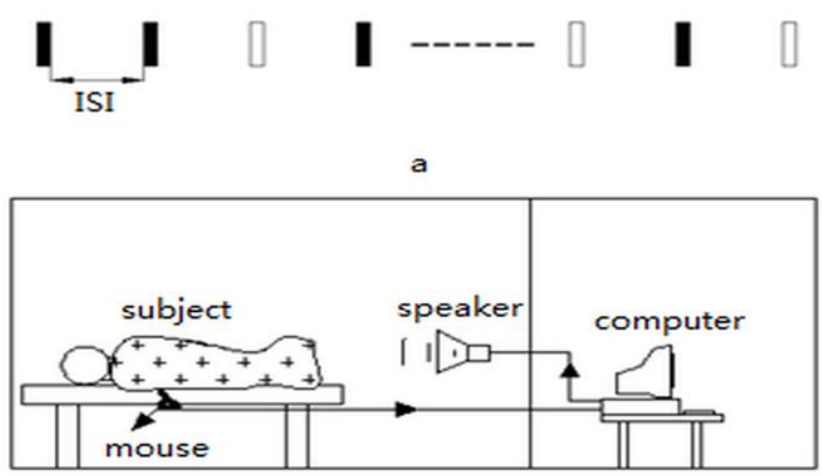

b

Figure 1. ISI and testing devices. (a) The inter-stimulus interval (ISI) was constant during the same testing session in order to satisfy with time series analysis. During the first two night testing session, ISI was 2 seconds and 3 ,

4 or 5 seconds was chosen in following days.(b) There were powered speakers and a mouse connected with the computer which was outside the testing room.

TABLE I. STIMULI RESPONSE CODING

\begin{tabular}{c|c|c|c}
\hline Stimuli & $\begin{array}{c}\text { Correct } \\
\text { response }\end{array}$ & $\begin{array}{c}\text { No } \\
\text { response }\end{array}$ & $\begin{array}{c}\text { False } \\
\text { response }\end{array}$ \\
\hline Signal A & $\mathrm{x}_{\mathrm{n}}$ & $\infty$ & $-\mathrm{x}_{\mathrm{n}}$ \\
\hline Signal B & $\mathrm{x}_{\mathrm{n}}$ & $\infty$ & $-\mathrm{x}_{\mathrm{n}}$ \\
\hline
\end{tabular}

Set of time series of behavioral response. The subscript $\mathrm{n}$ represents the number of stimuli occurring order.

There are three kinds of behavioral responses when an alternative stimulus is presented such as correct response, false response, and no response. In order to facilitate the analysis following, three kinds of responses are coding as following: correct response coding positive, false response coding negative and no response coding $\infty$ (shown in table 1).

Then, the reciprocal of behavioral responses is calculated, we get table 2 . Based on the new coding shown in table 2, all the behavioral response data in a testing session can be used to analysis. All the data for each participant were assessed with Eviews 6.0.

TABLE II. Stimuli response coding

\begin{tabular}{c|c|c|c}
\hline Stimuli & response & No response & False response \\
\hline Signal A & $\frac{1}{\mathrm{x}_{\mathrm{n}}}$ & 0 & $-\frac{1}{\mathrm{x}_{\mathrm{n}}}$ \\
\hline Signal B & $\frac{1}{\mathrm{x}_{\mathrm{n}}}$ & 0 & $-\frac{1}{\mathrm{x}_{\mathrm{n}}}$ \\
\hline
\end{tabular}




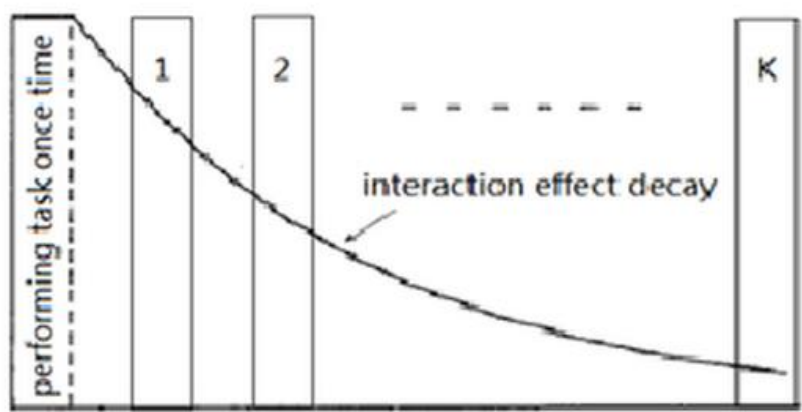

time

Figure 2. Decline time of interaction. The numbers $1,2,3 \ldots n$ present stimuli in turn. So, the length of the decline time of interaction can be approximately represented as $\mathrm{k} \times \mathrm{ISI}$ if the interaction decrease to zero near $\mathrm{k}$ stimuli presented time point.

\section{Algorithms of Extracting Decline Effect}

In the sound discrimination task, ISI is constant during the same testing session. We assign number $1,2,3 \ldots \mathrm{n}$ to the presented stimuli in turn. So, the length of the decline time of interaction can be approximately represented as kxISI if the interaction decrease to zero near $\mathrm{k}$ stimuli presented time point (See fig 2). Then, the behavioral response for first stimulus will affect subsequently behavior such as response for stimulus 2, 3 until $\mathrm{K}$.

Based on the observations transformation time-series data (written as yn) above (see table 3 row 1), these relationship can be listed in row 1. Similarly, this relationship exists among data $\mathrm{y} 2, \mathrm{y} 3, \mathrm{y} 4 \cdots \cdots, \mathrm{yk}+1$ (see table 3 row 2). And by analogy same relationship is among other rows data listed in table 3 .

In other words, column 1 affects each column subsequently. It indicates that column 1 correlate with column 2, 3 till $\mathrm{k}$ and this correlation relationship is represent with values of autocorrelations.

For example, for Lag 1, the second observation is paired with the first; the third observation is paired with the second, and so on, until the last observation is paired with the second from the last observation.

TABLE III.

AUTOCORRELATIONS AND PARTIAL AUTOCORRELATION

\begin{tabular}{c|c|c|c|c|c}
\hline Time & \multicolumn{5}{|c}{ Lag } \\
\hline & & 1 & 2 & $\cdots$ & $\mathrm{k}-1$ \\
\hline 1 & $\mathrm{Y}_{1}$ & $\mathrm{Y}_{2}$ & $\mathrm{Y}_{3}$ & $\cdots$ & $\mathrm{Y}_{\mathrm{k}}$ \\
\hline 2 & $\mathrm{Y}_{2}$ & $\mathrm{Y}_{3}$ & $\mathrm{Y}_{4}$ & $\cdots$ & $\mathrm{Y}_{\mathrm{k}+1}$ \\
\hline & $\vdots$ & $\vdots$ & $\vdots$ & $\vdots$ & $\vdots$ \\
\hline $\mathrm{n}$ & $\mathrm{Y}_{\mathrm{n}}$ & $\mathrm{Y}_{\mathrm{n}+1}$ & $\mathrm{Y}_{\mathrm{n}+2}$ & $\cdots$ & $\mathrm{Y}_{\mathrm{n}+\mathrm{k}-1}$ \\
\hline
\end{tabular}

If we now calculate the correlation between these paired observations, we will have calculated the Lag 1 autocorrelation. We should note that anyone of the autocorrelation of paired observations include all the interaction effect between them. Such as autocorrelation coefficient of lag 2 contains first and second time response interaction effect. In other words, autocorrelation coefficient of lag 2 reflects the mixed effect of first and second time response interaction effect. It imply that autocorrelation coefficient reflect the mixed effect of all interaction of current and before.

For autocorrelation of paired observations include all the interaction effect, and then excluding interaction effect except paired observations we will get the interaction decline effect. This pure interaction effect can be got by partial autocorrelation.

\section{RESULT}

\section{A. Participant Characteristics}

The subjects comprised 5 females and 2 males, with a mean age of 19.14 years $(\mathrm{SD}=0.69)$. The average PSQI global score was $8.86(\mathrm{SD}=0.9)$. During the test sessions, all subjects fell asleep except one subject in one session. This session was excluded from later analysis. The mean sleep onset latency was 8.31minutes ( $\mathrm{SD}=4.61$ ).

\section{B. The Reciprocal of Reaction Time Time-Series}

Based on data transformation of table 1 and table 2, we get reciprocal time-series of reaction time (See fig 3). It shows two morphological stages in reciprocal time-series. During the stage $\mathrm{B}$, the subjects make no response to any audio stimuli. The data collected in this stage will not be analyzed. Only the data collected in stage A is used to analysis.

Generally, 50 or more observations data provide reasonably accurate estimates in time series analysis of single subject[29-31]. The data being discussed later are satisfied with this condition.

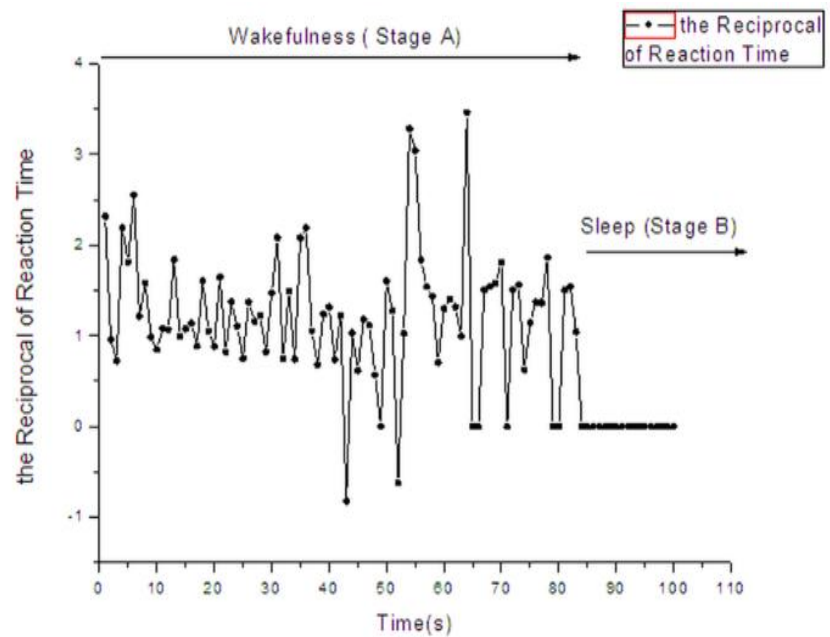

Figure 3. reciprocal time-series of reaction time. It shows two morphological stages in reciprocal time-series. The data collected in stage A is used to analysis. While during the stage B, the subjects make no response to any audio stimuli. So, the data collected there will not be analyzed. 


\section{Autocorrelation and Partial Autocorrelation of the Reciprocal of Reaction Time Time-Series}

Fig.4, fig.5 and fig.6 show the autocorrelation and partial autocorrelation of an insomniac's reciprocal of

\begin{tabular}{|c|c|c|c|c|c|c|}
\hline Autocorrelation & Partial Correlation & & $A C$ & PAC & Q-Stat & Prob \\
\hline 响 & 吅 & 1 & 0.168 & 0.168 & 21.975 & 0.000 \\
\hline 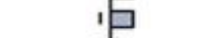 & 16 & 2 & 0.158 & 0.133 & 41.396 & 0.000 \\
\hline 10 & 11 & 3 & 0.030 & -0.016 & 42.109 & 0.000 \\
\hline 10 & 他 & 4 & 0.098 & 0.078 & 49.577 & 0.000 \\
\hline 1日 & 10 & 5 & 0.117 & 0.094 & 60.212 & 0.000 \\
\hline 19 & 1,1 & 6 & 0.056 & 0.003 & 62.701 & 0.000 \\
\hline 19 & 121 & 7 & 0.066 & 0.032 & 66.145 & 0.000 \\
\hline 12 & 10 & 8 & 0.069 & 0.046 & 69.854 & 0.000 \\
\hline 15 & 19 & 9 & 0.083 & 0.043 & 75.305 & 0.000 \\
\hline 15 & 1,1 & 10 & 0.043 & -0.001 & 76.752 & 0.000 \\
\hline 1$] 1$ & id & 11 & 0.002 & -0.031 & 76.754 & 0.000 \\
\hline 10 & 11 & 12 & 0.027 & 0.014 & 77.349 & 0.000 \\
\hline 101 & d & 13 & -0.034 & -0.058 & 78.283 & 0.000 \\
\hline 10 & 10 & 14 & 0.039 & 0.030 & 79.468 & 0.000 \\
\hline 11 & 1 & 15 & -0.013 & -0.018 & 79.610 & 0.000 \\
\hline id & di & 16 & -0.040 & -0.057 & 80.850 & 0.000 \\
\hline 11 & 11 & 17 & -0.012 & 0.003 & 80.969 & 0.000 \\
\hline 11 & 11 & 18 & -0.016 & -0.003 & 81.160 & 0.000 \\
\hline 11 & 11 & 19 & 0.011 & 0.010 & 81.263 & 0.000 \\
\hline $1 / 1$ & 101 & 20 & 0.024 & 0.037 & 81.712 & 0.000 \\
\hline
\end{tabular}

Figure 4. Autocorrelation and Partial Correlation. The autocorrelation and partial autocorrelation of an insomniac's reciprocal of reaction time series with ISI equaled to 2 seconds in different testing days.

\begin{tabular}{|c|c|c|c|c|c|c|c|c|}
\hline \multicolumn{2}{|c|}{ Autocorrelation } & \multicolumn{3}{|c|}{ Partial Correlation } & \multirow{2}{*}{$\frac{A C}{0.556}$} & \multirow{2}{*}{ 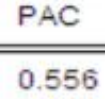 } & \multirow{2}{*}{$\begin{array}{l}\text { Q-Stat } \\
49.547\end{array}$} & \multirow{2}{*}{$\frac{\text { Prob }}{0.000}$} \\
\hline 1 & 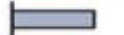 & 11 & ए & 1 & & & & \\
\hline 1 & 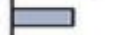 & 1 & 曰 & 2 & 0.409 & 0.144 & 76.515 & 0.000 \\
\hline 1 & 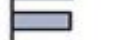 & 1 & 巨 & 3 & 0.388 & 0.166 & 100.86 & 0.000 \\
\hline 1 & $\square$ & ID & 1 & 4 & 0.234 & -0.092 & 109.78 & 0.000 \\
\hline 1 & 巨 & 10 & 1 & 5 & 0.147 & -0.036 & 113.33 & 0.000 \\
\hline 1 & 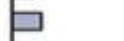 & 1 & 卢 & 6 & 0.200 & 0.127 & 119.95 & 0.000 \\
\hline 1 & 曰 & 1 & bi & 7 & 0.196 & 0.074 & 126.33 & 0.000 \\
\hline 1 & $\square$ & 1 & 曰 & 8 & 0.273 & 0.188 & 138.84 & 0.000 \\
\hline 1 & $\square$ & 1 & 曰 & 9 & 0.368 & 0.171 & 161.74 & 0.000 \\
\hline 1 & 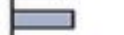 & 1 & 口 & 10 & 0.404 & 0.135 & 189.38 & 0.000 \\
\hline 1 & 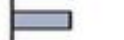 & 1 & Pi & 11 & 0.391 & 0.056 & 215.54 & 0.000 \\
\hline 1 & $\square$ & 1 & 1 & 12 & 0.340 & -0.021 & 235.44 & 0.000 \\
\hline 1 & $\boxminus$ & 1 & 1 & 13 & 0.285 & 0.006 & 249.52 & 0.000 \\
\hline 1 & $\square$ & 1 & $p_{1}$ & 14 & 0.274 & 0.080 & 262.58 & 0.000 \\
\hline 1 & $\square$ & 1 & pi & 15 & 0.262 & 0.088 & 274.61 & 0.000 \\
\hline 1 & 曰 & 1 & 1 & 16 & 0.205 & -0.021 & 282.08 & 0.000 \\
\hline 1 & 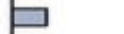 & 1 & pi & 17 & 0.241 & 0.051 & 292.45 & 0.000 \\
\hline 1 & $\ominus$ & 10 & 1 & 18 & 0.222 & -0.057 & 301.32 & 0.000 \\
\hline 1 & 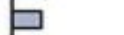 & 10 & 1 & 19 & 0.203 & -0.038 & 308.75 & 0.000 \\
\hline 1 & $\boxminus$ & 1 & 1 & 20 & 0.234 & 0.011 & 318.76 & 0.000 \\
\hline
\end{tabular}

Figure 5. Autocorrelation and Partial Correlation. The autocorrelation and partial autocorrelation of an insomniac's reciprocal of reaction time series with ISI equaled to 4 seconds in different testing days. 


\begin{tabular}{|c|c|c|c|c|c|c|}
\hline Autocorrelation & Partial Correlation & & $A C$ & PAC & Q-Stat & Prob \\
\hline 111 & $1 / 1$ & 1 & -0.019 & -0.019 & 0.0537 & 0.817 \\
\hline $1 \mathrm{~h}$ & $1 / 1$ & 2 & 0.040 & 0.040 & 0.2983 & 0.861 \\
\hline 10 & 15 & 3 & -0.044 & -0.043 & 0.5945 & 0.898 \\
\hline 1$] 1$ & 1 & 4 & 0.020 & 0.017 & 0.6541 & 0.957 \\
\hline 101 & 1 & 5 & -0.051 & -0.047 & 1.0546 & 0.958 \\
\hline 111 & 131 & 6 & 0.038 & 0.034 & 1.2852 & 0.972 \\
\hline 111 & $1 / 1$ & 7 & 0.031 & 0.038 & 1.4405 & 0.984 \\
\hline 101 & 101 & 8 & -0.058 & -0.065 & 1.9825 & 0.982 \\
\hline 101 & 101 & 9 & -0.053 & -0.053 & 2.4247 & 0.983 \\
\hline 연 & 명 & 10 & -0.175 & -0.175 & 7.3361 & 0.693 \\
\hline 101 & if 1 & 11 & -0.074 & -0.083 & 8.2154 & 0.694 \\
\hline 101 & 101 & 12 & -0.072 & -0.068 & 9.0613 & 0.698 \\
\hline $1 \mathrm{bi}$ & $1 \mathrm{~h}$ & 13 & 0.048 & 0.028 & 9.4404 & 0.739 \\
\hline $1 \mathrm{p}$ & 1 & 14 & 0.064 & 0.072 & 10.112 & 0.754 \\
\hline ין & 自 & 15 & 0.110 & 0.108 & 12.135 & 0.669 \\
\hline ין 1 & 16 & 16 & 0.105 & 0.132 & 13.973 & 0.601 \\
\hline 111 & $1 \mathrm{p}$ & 17 & 0.035 & 0.056 & 14.185 & 0.654 \\
\hline 101 & 101 & 18 & -0.058 & -0.068 & 14.752 & 0.679 \\
\hline 1$] 1$ & 18 & 19 & 0.005 & -0.029 & 14.757 & 0.738 \\
\hline 口 & ין 1 & 20 & 0.148 & 0.102 & 18.565 & 0.550 \\
\hline
\end{tabular}

Figure 6. Autocorrelation and Partial Correlation. The autocorrelation and partial autocorrelation of an insomniac's reciprocal of reaction time series with ISI equaled to 5 seconds in different testing days.

\section{DISCUSSION}

Based on the behavioral responses, sleep was defined as failure to respond to the faint auditory cue and the criterion for wakefulness is cognitive response to external stimulation[21]. In our experiment, the subject was asked to make behavioral response to audio stimuli. So, the behavioral criterion of the sleep was adopted. Based on this criterion, the brain was in sleep state during phased $\mathrm{B}$. And data of phase A reflected the cognitive interaction between cognitive tasks and unwanted thought.

Unwanted thought interacts with cognitive task, which will induce series of psychological effect having effect on the processing of falling asleep. For this interaction effect will not vanish immediately, the currently interaction effect will be hand on step by step until it decrease to zero level.

For the decline effect, the reciprocal time-series of reaction time will dependent. And the autocorrelation coefficient and partial autocorrelation coefficient all present the link among series of behavioral responses.

In our task, ISI is constant during the same testing session. So, the length of decline time can be approximately represented as $\mathrm{k} \times \mathrm{ISI}$ if the interaction reduces to zero near $\mathrm{k}$ stimuli presented time point (See fig 2).

The result shows that partial autocorrelation coefficient reduce slowly until zero and has a small correlation at lag 3 . It indicates that interaction effect reduces slowly until zero and the length of the declined tendency of interaction effect is less

Than 3 times ISI. For this test session, the ISI is 2 second, and then the interaction effect reduces to zero between the 4 and 6 seconds.

To test this prediction, in subsequent test session next day, the ISI is 4 seconds. For interaction effect reduces to near zero, and then the partial autocorrelation will be zero at lag 2. Surely as we expect, partial autocorrelation coefficient near zero lag 2(see fig 5). The same results are all yield in other insomniacs. Its shows that partial autocorrelation is reasonable in extracting decline effect.

Use this method, we invested the decline time of different insomniacs and found difference between them. This phenomenon may indicate that there are differences within insomniacs and another factor correlation with cognitive interaction effect. Obviously it needs new research design and intensive investigation which is beyond this paper.

In summary, we used a behavioral response task manipulated by computer to replace self-manipulating task to detect interaction effect between cognitive task and unwanted thought. And the reaction time time-series was got, which reflects changes of cognitive interaction. We proposed that the interaction effect will mix together for decline effect. Autocorrelation and partial autocorrelation analysis in time series method was adopted to extract mixed effect and decline effect. Such extracting is a potential candidate for investing correlation between cognitive load and interaction effect. And further test is necessary in subsequently studies.

\section{ACKNOWLEDGMENT}

Thank you to all the participants and co-authors. This research was financially supported by Social Science Foundation of The Chinese Education Commission grant 15609540 .

\section{REFERENCES}

[1] Nelson, J. and A.G. Harvey, Pre-sleep imagery under the microscope: a comparison of patients with insomnia and good sleepers. Behaviour Research and Therapy, 2003. 41(3): p. 273-284. 
[2] Harvey, A.G., Pre-sleep cognitive activity in insomnia: A comparison of sleep-onset insomniacs and good sleepers British Journal of Clinical Psychology, 2000. 39: p. 275-286.

[3] Espie, C.A., D.N. Brooks, and W.R. Lindsay, An evaluation of tailored psychological treatment of insomnia. Journal of Behavior Therapy and Experimental Psychiatry, 1989. 20(2): p. 143-153.

[4] Lichstein, K.L. and T.L. Rosenthal, Insomniacs' perceptions of cognitive versus somnatic determinants of sleep disturbance Journal of Abnormal Psychology, 1980. 89: p. 105-107.

[5] Geer, J.H. and E.S. Katkin, Treatment of insomnia using a variant of systematic desenstization: A case report. Journal of Abnormal Psychology, 1966. 71: p. 161-164.

[6] DE ZAMBOTTI, M., et al., Sleep onset and cardiovascular activity in primary insomnia. Journal of Sleep Research, 2011. 20(2): p. 318-325.

[7] Cincotta, A.L., et al., The effects of a mindfulness - based stress reduction programme on pre - sleep cognitive arousal and insomnia symptoms: a pilot study. Stress and Health, 2011. 27(3): p. e299-e305.

[8] Ansfield, M.E., D.M. Wegner, and R. Bowser, Ironic effects of sleep urgency. Behaviour Research and Therapy, 1996. 34: p. 523-531.

[9] Gross, R.T. and T.D. borkovec, The effects of a cognitive intrusion manipulation on the sleep onset latency of good sleepers. Behav. Ther., 1982. 13: p. 112-116.

[10] Field, T., Romantic Breakups, Heartbreak and BereavementRomantic Breakups. Psychology, 2011. 2(4): p. 382-387.

[11] Jansson-Fröjmark, M., et al., Psychometric properties of an insomniaspecific measure of worry: The anxiety and preoccupation about sleep questionnaire. Cognitive behaviour therapy, 2011. 40(1): p. 65-76.

[12] Carney, C.E. and J.D. Edinger, Sleep-Related Cognitive Processes, in Insomnia and Anxiety. 2010, Springer. p. 99-108.

[13] Harvey, A.G. and S. Payne, The management of unwanted pre-sleep thoughts in insomnia: distraction with imagery versus general distraction. Behaviour Research and Therapy, 2002. 40(3): p. 267-277.

[14] Salkovskis, P.M. and P. Campbell, Thought suppression induces intrusion in naturally occurring negative intrusive thoughts. Behaviour Research and Therapy, 1994. 32: p. 1-8.

[15] Haynes, S.N., A. Adams, and M. Franzen, The effects of presleep stress on sleep-onset insomnia. Journal of Abnormal Psychology, 1981. 90: p. 601-606.

[16] Bomyea, J. and N. Amir, The effect of an executive functioning training program on working memory capacity and intrusive thoughts. Cognitive therapy and research, 2011. 35(6): p. 529-535.
[17] Harrow, L. and E. Colin, Applying the quarter-hour rule: can people with insomnia accurately estimate 15 -min periods during the sleeponset phase? Journal of Sleep Research 2010. 19: p. 19-26.

[18] Tung, A., J.P. Lynch, and M.F. Roizen, Use of the BIS Monitor to Detect Onset of Naturally Occurring Sleep. J. Clin. Monitor. Comp., 2002. 17: p. 37-42.

[19] Ogilvie, R.D., The process of falling asleep. Sleep Med. Rev., 2001 5(3): p. 247-270.

[20] Ogilvie, R.D., et al., Behavioral, Event-Related Potential, and EEG/FFT Changes at Sleep Onset. Psychophysiology, 1991. 28: p. 54-64.

[21] Ogilvie, R.D., R.T. Wilkinson, and S. Allison, The detection of sleep onset: Behavioral, physiological, and subjective convergence. Sleep, 1989. 12(5): p. 458-474.

[22] Ogivie, R.D. and R.T. Wilkinson, Behavioral versus EEG-based monitoring of all-night sleep/wake patterns. Sleep, 1988. 11(2): p. 139-155.

[23] Kushikata, T., et al., The effects of neuropeptide $\mathrm{S}$ on general anesthesia in rats. Anesthesia \& Analgesia, 2011. 112(4): p. 845-849.

[24] Wilson, D.L., et al., Subjective Reports Versus Objective Measurement of Sleep Latency and Sleep Duration in Pregnancy. Behavioral sleep medicine, 2013(ahead-of-print): p. 1-15.

[25] Kertesz, R.S. and K.A. Cote, Event-related potentials during the transition to sleep for individuals with sleep-onset insomnia. Behavioral sleep medicine, 2011. 9(2): p. 68-85.

[26] Kanh, D., E.F. Pace-Schott, and J.A. Hobson, Consciousness in waking and dreaming: The roles of neuronal oscillation and neuromodulation in determining similarities and differences. Neuroscience, 1997. 78: p. 13-38.

[27] Buysse, D.J., et al., The Pittsburg Sleep Qqulity Index: A new instrument for psychiatric practice and research. Psychiatry Research, 1989. 28: p. 193-213.

[28] Pelli, D.G., The Video Toolbox software for visual psychophysics: Transforming numbers into movies. Spatial Vision, 1997. 10(4): p. 437-442.

[29] Ljung, G.M. and G.E.P. Box, On a measure of lack of fit in time series models. Biometrika, 1978. 65: p. 297-303.

[30] Glass, G.V., V.L. Willson, and J.M. Gottman, Design and analysis of time series experiments. 1975, Boulder, CO: Colorado Associate University Press.

[31] Box, G.E.P. and P.D. A., Distribution of Residual Autocorrelations in Autoregressive-Integrated Moving Average Time Series Models. J. Am. Stat. Assoc., 1970. 65: p. 1509-1526. 